\title{
Tracer Microstructure in the Large-eddy Dominated Regime
}

\author{
R. T. PIERREHUMBERT \\ Department of Geophysical Sciences, University of Chicago, Chicago, IL 60637, USA
}

\begin{abstract}
We have re-examined the inertial range behavior of a passive scalar which is advected by a large-scale velocity field causing a cascade of tracer variance to small scales, where it is dissipated by diffusion. This has been done within the context of an idealized model based on mixing by a 2D area-preserving map alternating with a weak diffusion step; the model is a special case of the general advection-diffusion problem. Both freely decaying and forced equilibrium systems were considered. Our main interest in this concerns the validity of Batchelor's theory predicting a $k^{-1}$ tracer variance spectrum, but the tracer microstructure has been diagnosed in terms of concentration probability distribution functions, generalized dimensions of the dissipation field, structure functions, and cancellation exponents. 2D simulations carried out at $1024^{2}$ resolution show that $i$, the decaying case the evolution sectles into a 'fractal eigenmode' in which the variance decays exponentially with time at a rate dependent on the Lyapunov exponent but independent of the diffusion coefficient. Although the concentration pattern is self-similar with time, the power spectrum is not algebraic. Concentration PDFs have exponential tails. The dissipation field is not multifractal, and formally has $D_{q}=2$ for all $q$. The convergence of the squared-gradient PDFs under coarse-graining indicates some underlying fractal behavior, however, and we have introduced the notion of "fractal degree of freedom' systems to describe such entities. Cancellation exponents and structure functions were also considered, and have a self-similarity which is compatible with a non-intermittent behavior of the dissipation field. These matters have also been addressed for the equilibrium case. The main difference is that the power spectrum of concentration variance in equilibrium exhibits a power-law inertial range, though it is steeper than $k^{-1}$, but not as steep as $k^{-2}$. Other features are similar to the decaying case. Very high resolution simulations of the undiffused problem indicate that a $k^{-1}$ spectrum is approached asymptotically, but only at resolutions corresponding to $10^{6} \times 10^{6}$. The passive scalar behavior is compared and contrasted with the behavior of scalar pseudo-vorticity (an 'active scalar') in the family of generalized 2D turbulence models introduced elsewhere in this issue.
\end{abstract}

\section{INTRODUCTION}

There are two great spectra believed to prevail for passive scalars under the action of turbulent or random velocity fields. The first is the $k^{-5 / 3}$ Obukhov-Corrsin spectrum prevailing in inertial-range homogeneous isotropic turbulence when the viscous cutoff of the velocity field is comparable to or smaller than the diffusive cutoff of the tracer field. In this case, the tracer microstructure is created by advection by the $k^{-5 / 3}$ velocity field, which has infinite shears and can be expected to lead to crinkling of tracer isosurfaces even at short times. This spectrum appears to be uncontroversial and robust, and is readily observable in natural and laboratory experiments.

The other spectrum is the Batchelor $k^{-1}$ spectrum [1], which is expected to prevail when the tracer diffusivity is so small that the smallest scales in the tracer field are smaller than the viscous cutoff of the velocity field. In this case, the tracer microstructure is created by long-term rearrangement by a smooth velocity field which, in Batchelor's theory is characterized by a single dominant timescale. To the best of our knowledge, this spectrum has never been unambiguously observed in laboratory experiments. The same problem has bedeviled the related $k^{-1}$ enstrophy spectrum of 2D turbulence. One begins to suspect that there may be something fundamentally wrong with Batchelor's argument. 
The essence here is the nature of mixing by an organized velocity field, and as such comes under the general heading of 'chaotic advection.' If Batchelor's argument is to work at all, it should work for chaotic mixing by simple flow fields. At the same time, the nature of microstructure created by chaotic mixing and small-scale diffusion is of considerable interest in its own right, and has many applications to naturally occurring flows [2-5]. There is also a great deal of current interest in more sophisticated measures of tracer variability than the spectrum: fractals, multifractals, probability distribution functions (PDFs) and cancellation exponents, e.g. refs $[6,7]$.

The general problem of mixing by an organized, smooth velocity field can be qualitatively captured by the action of area-preserving maps in two dimensions. Our overriding theme is to show how such maps with an interposed dissipative step can be used to probe the critical questions in a computationally tractable way. The model problem is described in Section 2. Freely decaying behavior is explored in Section 3, which includes a lengthy digression on the general subject of structure functions, cancellation exponents and generalized dimensions. Statistical equilibrium solutions are described in Section 4. Some extremely high resolution spectra for the undiffused case are given in Section 5 . Our principal conclusions are discussed in Section 6.

\section{DESCRIPTION OF THE MAP}

In this paper we explore mixing induced by the map

$$
\begin{array}{ll}
x_{n+1}=x_{n}+a \sin \left(y_{n}+\phi_{n}\right) & (\bmod 2 \pi) \\
y_{n+1}=y_{n}+\cos \left(x_{n+1}+\phi_{n}\right) & (\bmod 2 \pi)
\end{array}
$$

where $\phi_{n}$ is a random variable uniformly distributed over $[0,2 \pi]$. The randomization of the phase is introduced so as to break up invariant tori. Such tori are known to provoke very complicated scaling behavior in the stretching statistics, via the tendency of orbits to stick near tori or to become trapped behind cantori, e.g. ref. [8]. Conventional wisdom has it that such tori are uncharacteristic of real fluid turbulence, where the aperiodic flows make it more difficult for invariant structures to exist. In light of the prevalence of coherent structures in turbulence, we are not convinced of the validity of this assumption, but we adopt the randomized map (1) anyway, because its statistics are easier to study.

Because of the complete scrambling of the phase, each individual orbit undergoes an uncorrelated random walk on the torus. However, because the same $\phi_{n}$ is used at each iteration throughout the phase space, interesting spatial correlations nevertheless arise from (1). The most important characterization of the map is through its stretching statistics. The Jacobian of the map is

$$
J=\left(\begin{array}{cc}
1 & a \cos \left(y_{n}+\phi\right) \\
-\sin \left(x_{n+1}+\phi\right) & {\left[1-a \cos \left(y_{n}+\phi\right) \sin \left(x_{n+1}+\phi\right)\right]}
\end{array}\right)
$$

which has eigenvalues

$$
e^{\lambda}=\gamma \pm V\left(\gamma^{2}-1\right), \quad \text { where } \quad \gamma=1-\frac{1}{2} a \cos \left(y_{n}+\phi\right) \sin \left(x_{n+1}+\phi\right)
$$

where $\lambda$ is the exponential stretching rate over one iteration. Note $\gamma \in\left[1-\frac{1}{2} a, 1+\frac{1}{2} a\right]$. For any phase and any value of $a$, there is always a part of the domain near which the trajectories separate exponentially. Because of averaging over the spectrum of strains, the long-time Lyapunov exponent is considerably lower than the maximum given by (3). For maps without invariant tori it is known that the finite-time ( $n$-iteration) Lyapunov exponents have a Gaussian PDF, with width decreasing like $n^{-1 / 2}$ [9]. We have verified this 
Table 1. Finite time Lyapunov exponents

\begin{tabular}{lcc}
\hline Iterations & Mean $(\lambda)$ & Std. Dev $(\lambda)$ \\
\hline 10 & 0.57 & 0.20 \\
20 & 0.53 & 0.14 \\
40 & 0.50 & 0.10 \\
\hline
\end{tabular}

property directly for the map (1), by accumulating multiplications of $J$ over ensembles of orbits. For $a=4$, which is the parameter used throughout the rest of this paper, the mean and standard deviation of the exponential stretching rates for various $n$ are given in Table 1.

There have been innumerable studies of mixing and tracer microstructure due to maps such as (1). The chief novel interest in the present work comes from incorporating the effects of weak diffusion, which removes tracer variance as it cascades to small scales. Within the context of discrete-time maps on the plane, this is accomplished in the following manner. We define a continuous concentration field $c(x, y)$ on the plane, and spatially discretize it to a regular grid so $c_{i j}=c\left(x_{i}, y_{j}\right)$. The map (1) is first used to rearrange $c(x, y)$, through mapping $(x, y)$ and re-interpolating to the grid. This iteration, which yields a new $c(x, y)$ is followed by the diffusion step:

$$
c_{i j}=(1-D) c_{i j}+\frac{1}{4} D\left(c_{(i+1) j}+c_{i(j+1)}+c_{(i+1) j}+c_{i(j-1)}\right)
$$

where $0<D<1$. On each iteration, this damps out the $2 \Delta x$ wave by a factor $(1-D)$. A generally similar approach has been employed by Ott et al. [10], and Du and Ott [11] to study diffusive effects on fast magnetic dynamos.

\section{FREELY DECAYING EVOLUTION}

Because of the introduction of dissipation, the variance of the tracer field $c_{n}(x, y)$ will decay to zero with time. The first property of interest is the rate of decay, and how the rate scales with diffusivity $D$. The variance decay for $D=0.5$ and $D=1$ is shown in Fig. 1 ; the initial condition for this case is $c(x, y)=\cos (x) \cos (y)$, which would not perceptibly

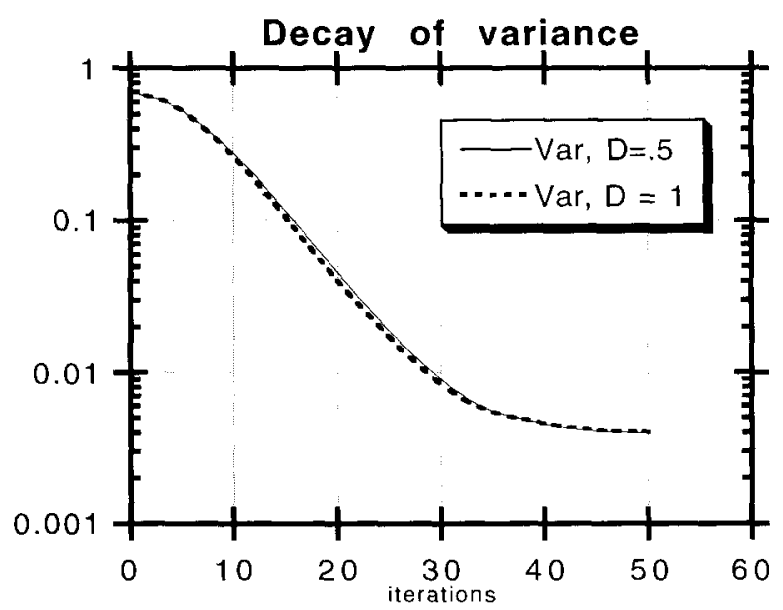

Fig. 1. Decay of variance for mixing by randomized sine-sine map, with two different values of diffusion $D$. 
decay over the time period shown, were it not for the exponential amplification of gradients caused by the chaotic mixing.

At long times, the variance reaches a small nonzero asymptotic value. This is an artifact of the numerical method, arising from a small variance source associated with interpolation errors in going between the map (1) and the discrete grid on which the diffusive step is carried out. Apart from this, the variance decay is independent of $D$, and has an exponential stage at intermediate times. We conjecture that the exponential stage would continue indefinitely were it not for discretization error; verification of this conjecture must await formulation of a more accurate discrete advection-diffusion scheme.

The diffusion-independent decay stage shows that the simple map can reproduce the classical inertial-range cascade to small scales, where variance can be dissipated. The independence of $D$, of course, comes from the fact that as $D$ gets smaller, the dissipation range simply moves to smaller scales. Further light can be shed on the decay properties through examination of the variance equation

$$
\frac{\mathrm{d}}{\mathrm{d} t}\left\langle c^{2}\right\rangle=-\kappa\left\langle|\nabla c|^{2}\right\rangle,
$$

where $\boldsymbol{K}$ is the molecular diffusivity. If $\lambda$ is the stretching rate experienced by a fluid element (roughly the typical Lyapunov exponent), then balancing strain against diffusion yields a filament scale $L=V(\kappa / \lambda)$, whence the gradient scales like $\delta c / L$, where $\delta c$ is the typical concentration gradient across a filament. Then, if $\delta c$ can be estimated as $\delta c=b$ $\sqrt{ }\left\langle c^{2}\right\rangle$ the variance decay rate becomes simply $b^{2} \lambda$, which is indeed independant of diffusivity. In the numerical experiments, the variance decays approximately like $\exp (-0.178 n)$. Using $\lambda=0.5$ from the Lyapunov exponent calculation, we infer $b=0.6$. The fact that $b$ is order unity indicates that the concentration values are well 'scrambled.' The typical jump across a filament is similar to what would be obtained by randomly picking values from a distribution covering the range prevailing over the entire domain. It can easily be shown that these results are not altered if the stretching rate is characterized by a distribution of different $\lambda$ instead of a unique value. As long as $\delta c$ is independent of $\lambda$, fluctuations in $\lambda$ only require replacing $\lambda$ in the above formulae by its mean value.

The exponential decay suggests that, following a transient adjustment stage, the concentration pattern settles into an 'eigenmode.' The amplitude decays, but the pattern is (in some statistical sense) time-invariant. A snapshot of the concentration eigenmode at $n=25$ is shown in Fig. 2. One is tempted to invoke the term 'fractal,' but it would perhaps be less prejudicial at this point to refer to it as a 'strange eigenmode.' The indication is, at any rate, that this 'eigenmode' has arbitrarily fine scales, that are limited only by the presence of diffusion. The behavior is similar to the 'horrible eigenvalue problems' discussed by Bayley [12]. There is a close analogy with the less exotic example of critical-line singularity in the shear instability problem. In that case, as viscosity approaches zero the eigenmodes show arbitrarily fine structure near the line where Doppler-shifted phase speed vanishes. Perhaps the 'strange eigenmodes' can profitably be viewed as a situation in which the 'critical line,' which is really just a form of resonance, becomes space-filling.

To flesh out the notion of a 'strange eigenmode' we need to quantify in what sense the 'eigenmode' is time-independent, by studying statistical characterizations of the spatial structure of the concentration field. The power spectrum is one of the most familiar characterizations of the spatial structure of a field. Power spectra at $n=10,20$ and 30 are shown on a log-linear plot in Fig. 3. All fields have been normalized to have unit variance, and the wavenumbers have been normalized such that $k=512$ corresponds to the $2 \Delta x$ wave. Notably, the spectra collapse onto the same curve for wavenumbers $>10$, indicating 


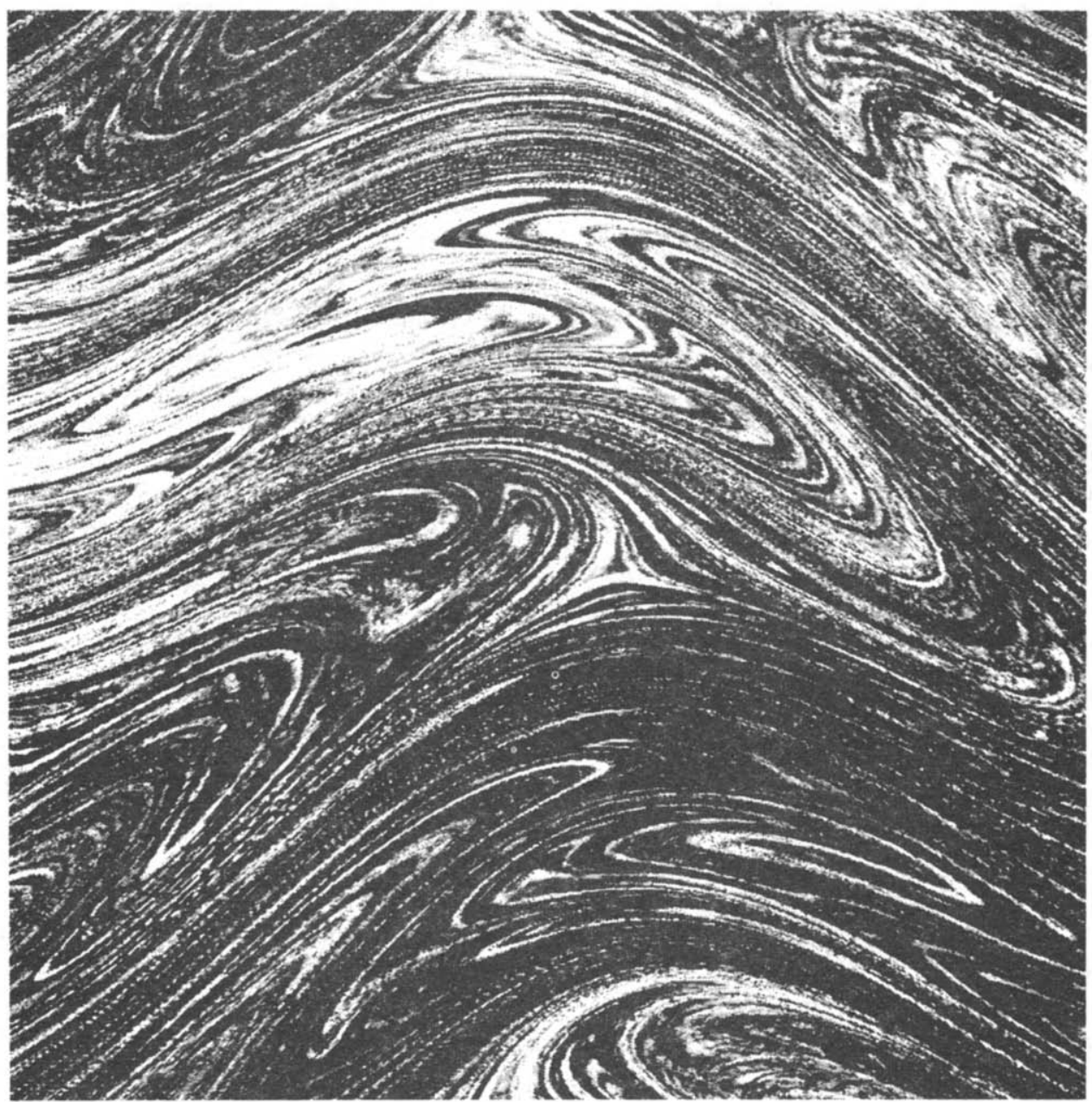

Fig. 2. Snapshot of concentration pattern for the decaying mode after 25 iterations.

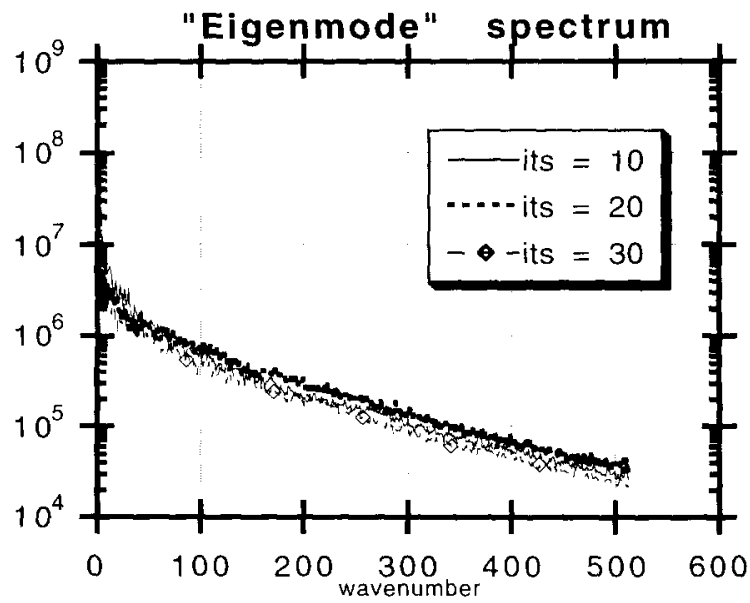

Fig. 3. Concentration power spectra of the decaying mode at $n=10,20,30$ and 250 (note linear-log axes). 
self-similar behavior in time. Further, the spectra do not have a power-law form $\left(k^{-1}\right.$ or otherwise) even at large scales that are not directly affected by dissipation. Rather, the spectra show exponential rolloff at constant rate for wavenumbers $>10$. Thus, the spectral behavior supports the notion that a (strange) eigenmode emerges, but also shows that Batchelor's argument leading to a $k^{-1}$ spectrum fails when where is not a sufficient resupply of variance from large scales. In fact, the possibility of a breakdown of this sort was hinted at in ref. [1]; it was remarked there that the $k^{-1}$ spectrum implies unbounded variance in the limit of small dissipation, whereas the variance is globally bounded for many physical means of maintaining concentration variance (e.g. introducing dye at a fixed concentration). Batchelor noted that something different must happen when the supply of variance (' $\theta^{2}$-stuff,' as he called it) from large scales is insufficient to fill out the $k^{-1}$ spectrum. Up to now, there have been no suggestions as to just what happens when the breakdown takes place. We conjecture that as the global limit of ' $\theta^{2}$-stuff' becomes much smaller than that needed to fill out the $k^{-1}$ spectrum to the dissipation range, the exponential behavior characteristic of the eigenmode takes over. We shall see in Sections 4 and 5, however, that there appear to be other factors at work that also tend to yield spectra steeper than $k^{-1}$.

The concentration PDF provides more detailed information about the tracer variability, though by itself it contains no information about spatial scales. Following the experimental work on temperature PDFs in turbulent convection (see ref. [13] and references therein) and the theoretical work by Sinai and Yakhot [7] and by Pumir et al. [14], there has been considerable interest in the mechanisms leading to non-Gaussian PDFs. In particular, the appearance of exponential rather than Gaussian tails implies a relatively high probability of extreme events-parcels surviving a long time without mixing much with their environment. PDFs of concentration at $n=25$ are shown in Fig. 4(a). The PDF is self-similar for $n=10$ through 30 , which further supports the notion that the decaying field is an eigenmode. The PDF is unimodal, indicating efficient mixing between high and low concentrations present in the initial field. While the profile is Gaussian for small to intermediate fluctuations, the large-fluctuation tail is markedly exponential. The decaying 'eigenmode' we have studied is a perfect match for the assumptions of Sinai and Yakhot [7]; these authors have shown that non-Gaussian tails arise from correlations between the concentration field and its gradient, and that the advection-diffusion equation can generate such correlations on its own. We believe the decaying mode of the advection-diffusion

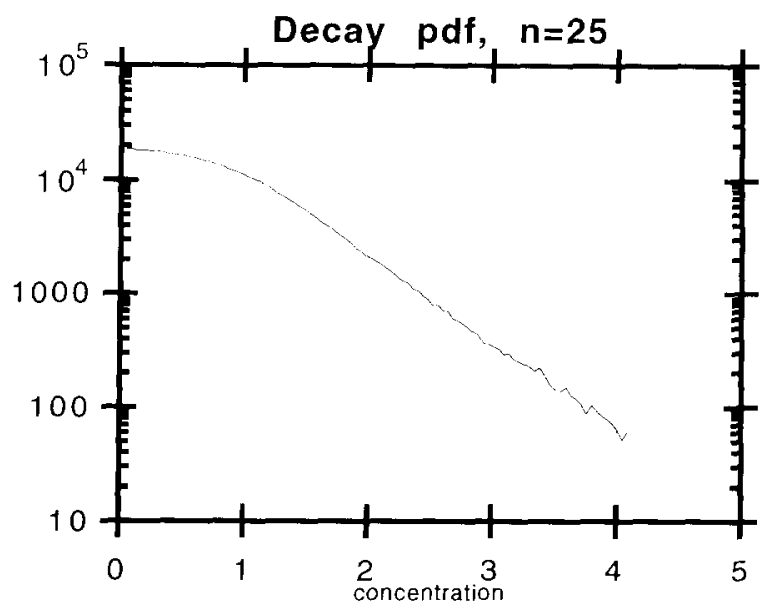

Fig. 4(a). PDF of concentrations for the decaying mode at $n=25$ (linear $-\log$ plot). The PDF is symmetric about $c=0$, so only positive concentrations are shown. 
map is the simplest example to date of a system in which the Sinai-Yakhot theory works. In the next section, however, we will see that the theory can break down in the forced/dissipated equilibrium situation.

The concentration field itself has bounded variations and therefore cannot show multifractal scaling. Indeed, a calculation of the behavior of the concentration PDFs under various degrees of spatial smoothing revealed no interesting scaling behavior. The gradient fields, however, can have singularities (at long time, in the limit of small diffusion), and therefore can conceivably show multifractal scaling. The PDFs of gradients also contain information about the spatial arrangement of the concentration fluctuations. To address this question, we normalized the $n=25$ field to have unit variance, computed the squared-gradient of this field, subjected the resulting field to various degrees of smoothing, and computed the PDFs. Results are shown in Fig. 4(b). These curves are fit almost exactly by the lognormal distribution:

$$
P(g)=\frac{1}{g \sigma \sqrt{ } \pi} \exp \left[-\left(\frac{\log \left(g / g_{1}\right)}{\sigma}\right)^{2}\right],
$$

where $g$ is the squared-gradient. The parameters of the fit are given in Table 2.

The $q$ th moment of the distribution (6a) is

$$
\zeta_{q}=g_{1}{ }^{q} \exp \left(\sigma^{2} q^{2} / 4\right)
$$

The behavior of $g_{1}$ and $\sigma$ under smoothing thus determine the scaling properties of the gradient field. If we assume the $\log$ of the gradient to be a random field of some sort

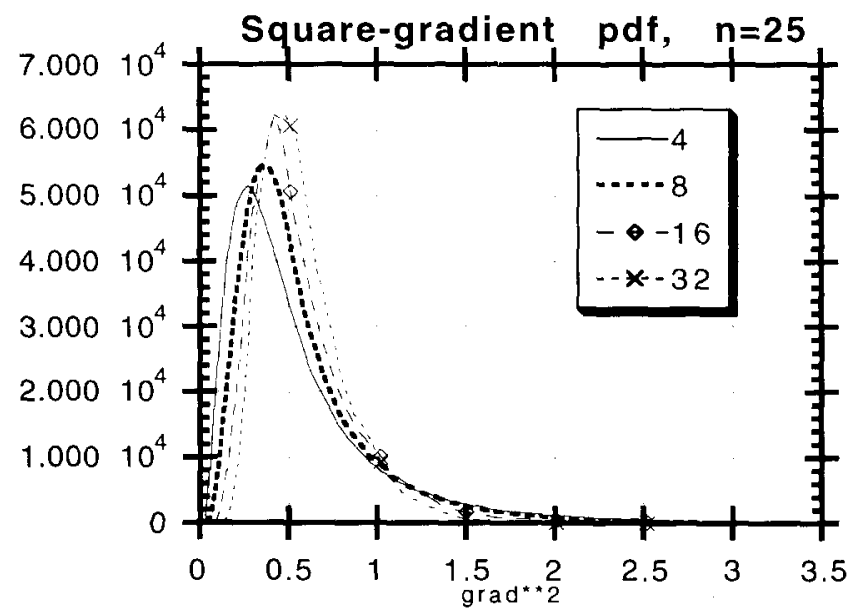

Fig. 4(b). Squared-gradient PDF as function of smoothing for $n=25$ in the decaying case. Abscissa is fixed for all smoothings, and has not been renormalized.

Table 2 .

\begin{tabular}{rll}
\hline Box size & $g_{1}$ & $\sigma$ \\
\hline 4 & 2.32 & 1.03 \\
8 & 2.13 & 0.80 \\
16 & 2.00 & 0.67 \\
32 & 1.9 & 0.57 \\
64 & 1.9 & 0.24 \\
128 & 1.9 & 0.16 \\
\hline
\end{tabular}


(related to the distribution of Lyapunov exponents), then the scaling of $\sigma$ gives the effective number of degrees of freedom within a box of side $m$. If the field were $2 \mathrm{D}$ white noise, then the number of degrees of freedom is $m^{2}$, so we would have $\sigma=m^{-1}$. If the field were constant on each of an array of space-filling thin filaments, but randomly distributed across filaments, the number of degrees of freedom would be $\mathrm{m}^{1}$, and so we would have $\sigma=m^{-0.5}$. Other values of the exponent would be indicative of a 'fractal number of degrees of freedom.' However, power-law scaling of $\sigma$ (with $g_{1}$ fixed) would not correspond to a multifractal. For the field to be multifractal, $\zeta_{q}$ must be a power-law in $m$, the exponent of which determines the generalized dimension $D_{q}$ for the squared-gradient measure. According to (6b), then, multifractal scaling requires $\sigma^{2}=-a \log (m / M)$. If $\sigma^{2}=m^{-\gamma}$ instead, the scaling of $\zeta_{q}$ treated as a power-law is asymptotically trivial, and we have $D_{q}=D$, where $D$ is the dimension of the whole space. It is interesting to note that we obtain a non-intermittent field $\left(D_{q}=D\right)$ in the limit of a random field which is nowhere-differentiable as well as in the limit a smooth field. Smooth fields and random fields are opposite extremes of non-intermittency, with multifractals lying in between. Clearly, when $D_{q}=D$, the multifractal formalism fails to characterize the geometry of the field. On the other hand, the exponent $\gamma$ does contain important geometric information. We introduce the term 'fractal degree of freedom' (FDF) field for random fields that have a power law for $\sigma$ having non-integer $\gamma$.

For the data in Table 2, the width parameter exhibits the scaling $\sigma \approx m^{-0.29}$, and is decidedly different from the behavior required of a multifractal. Thus, the squared-gradient field for this advection-diffusion problem appears to be an FDF random field rather than a multifractal. Since $\gamma=0.58$, the spatial structure of concentration gradients exhibits more long-range order than even the filamentary model would imply, as filaments yield $\gamma=1$. This suggests considerable correlations of gradient values amongst bundles of filaments, which retard the convergence of the coarse-grained gradient to its mean value.

Multifractal scaling of gradients of undiffused tracers has been predicted by Varosi et al. [15] but, apart from the nondiffusive limitation, there are some important differences between the approach of Varosi et al. and that employed here. In their theoretical formula linking the generalized dimension spectrum to the PDF of Lyapunov exponents, Varosi et al. employ a variable box-size cover designed to resolve each filament individually. Boxes in the cover do not contain an ensemble of filaments. This makes it possible to compute multifractal properties of the field in terms of the PDF of Lyapunov exponents, but in the process, all information about the spatial arrangement of the filaments is lost; it makes no difference whether the filaments are straight or curled, or whether the high-gradient filaments are segregated or randomly distributed. Our fixed-box calculation, in contrast, retains the geometric information. Most analysis of experimental data would use a cover that is more like the uniform-sized cover we employ in our analysis. In such a case, we would predict that the squared-gradient field is an FDF random field rather than a multifractal, for concentration fluctuations dominated by advection having a characteristic timescale. This comment notwithstanding, the relation of our results to results of the type of Varosi et al. requires further exploration.

The scaling properties of squared-gradient fields provide a lot of interesting information about the arrangement of gradients, but lose all information relating to the sign of the gradients; these characterizations would be invariant under multiplication of the underlying signed gradient by an arbitrary field taking on the values +1 or -1 . Worse still, in two dimensions we can rearrange not only the sign of the gradients but also the directions without altering the scaling properties of the squared-gradient fields. The lost information is of great importance to many physical applications, because it makes a great deal of difference whether gradients of like sign tend to congregate, or whether alternating signs 
occur in close proximity so that in the average they tend to cancel out. The problem, of course, arises from the fact that the operations of smoothing and taking the square do not commute.

Interest in matters related to sign-singularity and restoration of the missing information has arisen in a number of contexts recently. The classical way of characterizing the variability of a field $\theta(\mathbf{r})$ is through its set of structure functions:

$$
Z_{L}(q)=L^{-q}\left\langle|\theta(\mathbf{r}+L \hat{\mathbf{e}})-\theta(\mathbf{r})|^{q}\right\rangle
$$

where $L$ is a length and $\hat{\mathbf{e}}$ is a unit vector whose direction is immaterial in a statistically isotropic situation. Angle brackets represent a spatial average over the entire domain. The description in terms of $Z(q, L)$ is equivalent to a description in terms of the $\operatorname{PDF}_{L}(g)$ of the mean gradient

$$
g=\frac{|\theta(\mathbf{r}+L \hat{\mathbf{e}})-\theta(\mathbf{r})|}{L}
$$

since the PDF can be recovered from the set of moments and vice versa provided that the tails of the PDF are steeper than algebraic. The structure functions retain information about the sign of the bare gradient, because the averaging over $L$ is performed before the absolute value is taken. Scaling laws for velocity structure functions have long played a key role in characterization of turbulence, and through application of Kolmogorov's second hypothesis can be linked to the generalized dimensions of the energy dissipation field (see ref. [16] and references therein). If $Z_{L}(q)$ has a power-law behavior in $L$, the exponents behave very much like generalized dimensions, and the relation between $Z$ and $\Pi$ obeys a thermodynamic formalism essentially identical to that relating $D_{q}$ and $f(\alpha)$ for multifractals. This has been exploited in analysis of turbulence data by Du et al. [17] for analysis of magnetic fields and turbulent velocity fields, and by Marshak et al. [18] and Davis et al. [19] in characterization of a variety of cloud-related atmospheric quantities. It has recently become clear that the structure functions are in fact intimately related to the generalized dimensions $D_{q}$ of the gradient-magnitude field $|\nabla \theta|$, provided one additional bit of information-the cancellation exponent is specified. Cancellation exponents were first introduced by Ott et al. [10] and Du and Ott [11], and have been subsequently explored in more depth by Du et al. [17] and by Vainshtein et al. [20]. Our discussion in the following is based loosely on these works, except that we have made a few straightforward changes to allow us to deal with concentration gradients rather than magnetic fields or vorticity fields.

Let $\bar{c}(\mathbf{r})$ be the concentration field smoothed spatially over lengthscale $L$. We imagine the smoothing to be carried out by spatially averaging over a shape of size $L$ (e.g. a square or a circle) centered on $\mathbf{r}$. Now define the moments

$$
Z_{L}(q)=\left\langle|\nabla \bar{c}|^{q}\right\rangle \text {. }
$$

In one dimension, this is identical to the structure function (7). In two or more dimensions, it is similar to, but not identical to, a structure function. The difference arises because the mean gradient $g$ in (8) results from integrating the gradient along a line, whereas the $2 \mathrm{D}$ smoothing operator in (9) implies

$$
\nabla \bar{c}=\frac{1}{A} \int_{\Gamma} c(\mathbf{r})(-\hat{x} \mathrm{~d} y+\hat{y} \mathrm{~d} x)
$$

where $A$ is the area of the smoothing region and $\Gamma$ is its boundary. For a square-averaging region, this yields the spatial difference between the one-dimensional averages of $c$ along opposing edges of the square, rather than between the values of $c$ itself. The extra average 
is what keeps $Z_{L}$ in (9) from being identical to the classical structure function. In some cases, it may be possible to relate the two structure functions by employing auxiliary assumptions about the field. Henceforth, by 'structure function' we will mean (9). The corresponding PDF of the mean gradient will be referred to as the 'structure PDF.'

If $Z_{L}(q)$ has a power-law dependence on $q$, then the spectrum of cancellation exponents $\kappa_{q}$ is defined by

$$
Z_{L}(q) \approx L^{-\kappa_{q}-(q-1) D}
$$

This formula differs somewhat in appearance from that used by Du et al. [17], because we have defined $Z_{L}$ in terms of an average instead of a sum, but it is in fact equivalent. $Z_{L}(2)$ gives the scaling of the smoothed variance field, and so $\kappa_{2}$ is directly related to the power spectrum. Specifically, if the power spectrum of $c$ has the scaling $k^{-\beta}$, then $Z_{L}(2) \approx L^{\beta-3}$ whence

$$
\kappa_{2}=3-\beta-D \text {. }
$$

It is a very remarkable result, due to Du et al. [17], that the $\kappa_{q}$ are not all independent, and in fact can be determined given knowledge of $\kappa_{1}$ and the generalized dimensions $D_{q}$ for the gradient-magnitude field. This means the $\kappa_{1}$ alone is sufficient to characterize the cancellation, and to permit reconstruction of structure functions from knowledge of $D_{q}$. The remarkable relationship is:

$$
\kappa_{q}=q \kappa_{1}-(q-1) D_{q}
$$

whence

$$
Z_{L}(q) \approx L^{-q \kappa_{1}-(q-1)\left(D-D_{q}\right)}
$$

This permits all the cancellation exponents to be obtained in terms of $D_{q}$ and the familiar power spectrum. It is interesting to note that the fractional integration approach in Tessier et al. [21] characterizes cloud-field variability by a $D_{q}$ for the gradient-magnitude field and a power spectrum $H$ for underlying field; the implication of (14) is that the fractionally integrated fields obtained from their procedure should have the correct structure function, despite the fact that only the behavior of the variance scaling $H$ is built into the construction.

From (12) and (13) it follows that $\kappa_{1}=\frac{1}{2}\left(D_{2}-D+3-\beta\right)$. The generalized dimension $D_{2}$ measures the intermittency of the dissipation field. For the Batchelor spectrum, $\kappa_{1}$ ranges from 1 for space-filling dissipation $\left(D_{2}=D=2\right)$ to 0 for dissipation concentrated on isolated points $\left(D_{2}=0\right)$. The Batchelor spectrum is thus compatible with both intermittent and non-intermittent dissipation fields, because differences in the intermittency can be offset by different degrees of cancellation. Intermittency is reflected in the behavior of the structure PDF, however. If the $|\nabla c|$ field is monofractal, with $D_{q}=D^{\prime}$ for all $q$, then (14) implies that the corresponding structure PDF is self-similar under rescaling of its argument by $L^{-\kappa_{1}-\left(D-D^{\prime}\right)}$. In the limit of a space-filling gradient field, so $D^{\prime}=D$, the renormalization factor is $Z_{L}(1)$ itself. Such behavior can be referred to as 'Kolmogorovian,' by analogy with a similar hypothesis made concerning the relation between velocity structure functions and the energy dissipation field in the K41 homogeneous turbulence phenomenology. On the other hand, if $|\nabla c|$ is a multifractal field the PDFs will not be self-similar.

We can estimate $Z_{L}(2)$ in terms of random field models of the signed quantity $\nabla c$, much as we did for the squared-gradient field. Consider the two-dimensional case. If $\nabla c$ is white noise distributed on filaments, then $Z_{L}(2) \approx L^{-1}$, whence $\beta=2$. This result provides an alternate derivation of the Saffman $k^{-2}$ spectrum [22], which arises from uncorrelated jumps of the value of a scalar across smooth curves. If $\nabla c$ is $2 \mathrm{D}$ white noise, then 
$Z_{L}(2) \approx L^{-2}$, and we have the Batchelor spectrum $\beta=1$. This provides a geometric interpretation of the Batchelor spectrum, as well as of spectra with steepness intermediate between the Batchelor and Saffman spectra. Intermediate spectra correspond to gradient structures that are more folded than filaments, yet not so much crinkled as to produce $2 \mathrm{D}$ white noise.

Because the concentration power spectrum for the decaying tracer case does not exhibit a well-defined power-law, one cannot unambiguously compute a cancellation exponent for this case. One can nonetheless compute the structure PDF and examine its scaling properties under averaging of $c$. In Fig. 4(c) we show the structure PDFs for various $m$ after 25 iterations. For each $m$, the argument of the PDF has been rescaled by the mean gradient $\langle|\nabla \bar{c}|\rangle$. The most important result is that the rescaled PDFs are almost exactly self-similar under variation of averaging length $m$. This is what would be expected for a non-intermittent gradient magnitude field with $D_{q}=D$ for all $q$. A further interesting feature is that the most probable gradient is nonzero. This demonstrates the existence of long-range correlations in the fields. If the smoothed gradient were behaving like the difference of two samples randomly chosen from the concentration PDF, the most probable gradient would instead be zero.

\section{EQUILIBRIUM PROBLEMS}

The preceding has provided a characterization of the tracer variability for the freely decaying case, and we have seen that the overall picture may be described in terms of a 'strange eigenmode.' In many situations of physical interest, however, the tracer variance is maintained against small-scale dissipation by large-scale forcing. In the present section, we will see how the introduction of such forcing alters the picture obtained in the decaying case.

We employ the following forcing protocol to maintain variance. Within a strip of width $2 \varepsilon$ centered on the line $y=\pi / 2$, we reset the concentration to +1 at each iteration; the corresponding strip centered on $y=-\pi / 2$ is reset to -1 . This kind of forcing is adopted rather than the more common (in numerical experiments only!) additive forcing $c \rightarrow c+F$

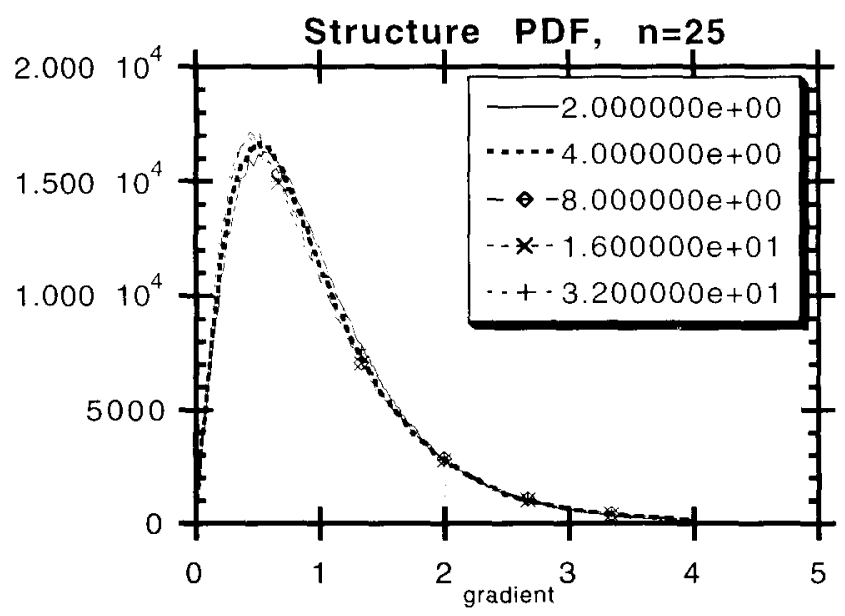

Fig. 4(c). Rescaled PDFs of magnitude of the gradient computed after various degrees of smoothing of the concentration field. Abscissa has been rescaled by the r.m.s. gradient for each curve. Note that this is the PDF for the gradient, rather than for the squared-gradient. The squared-gradient PDF peaks at zero gradient instead of a finite value. 
because we believe it to be more representative of the means by which concentration variance is maintained in most physical experiments. In particular, the 'resetting' forcing assures that the concentrations will remain in a bounded range $c \in[-1,1]$, so that the variance remains globally bounded. This is similar to the situation in which the tracer represents dye concentration, and the dye is introduced at fixed concentration from a nozzle, or by diffusion from a solid source at a boundary (whereupon concentration is maintained at saturation in a diffusive boundary layer). It is also similar to the situation prevailing for temperature in convection experiments. Fluid parcels in that case are reset to either the temperature $T_{1}$ of the hot lower boundary or $T_{2}$ of the cold upper boundary only when they are processed through the thin thermal-boundary layers near each plate. The PDF theory described by Sinai and Yakhot [7] neglects the dynamics of variance maintenance associated with this process, and so it will be of particular interest to see what concentration PDF emerges in the equilibrium case.

It should be noted that our forcing protocol destroys some small-scale variance within the thin strips, as well as maintaining the large-scale variance. Variance is free to cascade to small scales outside the strips, however. The rate at which the forcing protocol removes variance depends on the frequency with which parcels are processed through the forcing strips. Assuming ergodicity, a parcel has a high probability of passing through one of the strips after $\pi / 2 \varepsilon$ iterations. For small $\varepsilon$, this leaves ample time for exponential enhancement of gradients outside the strips. Similar considerations are of physical interest in the case of diffusive thermal or concentration boundary layers in laboratory experiments.

We turn first to the spectra, which have settled into an equilibrium after 25 iterates. The spectra for $n=25,50,250$ and 500 with $\varepsilon=0.1$ are shown in Fig. 5. Apart from some continued evolution in the dissipation range, the spectra have converged by $n=25$. These calculations were carried out with dissipation $D=0.5$. Notably, there is a clear power-law range. Equally notably, however, the spectrum is steeper than the $k^{-1}$ Batchelor spectrum. The best fit power-law for $k>20$ is $k^{-1.6}$. It thus appears that though this is a situation where Batchelor's argument ought to apply precisely, the tracer spectrum shows anomalous steepness. This phenomenon appears to be robust, extending well beyond passive tracers and mixing by iterated maps; anomalous steepness has been found in a family of fully nonlinear 2D turbulence models [22] in the range where large-eddy advection should dominate. In particular, the spectral shape for $\alpha=2$ is $k^{-1.4}$ and for $\alpha=3$ is $k^{-1.3}$. Is this anomalous steepness due to insufficient separation between the dissipation range and the

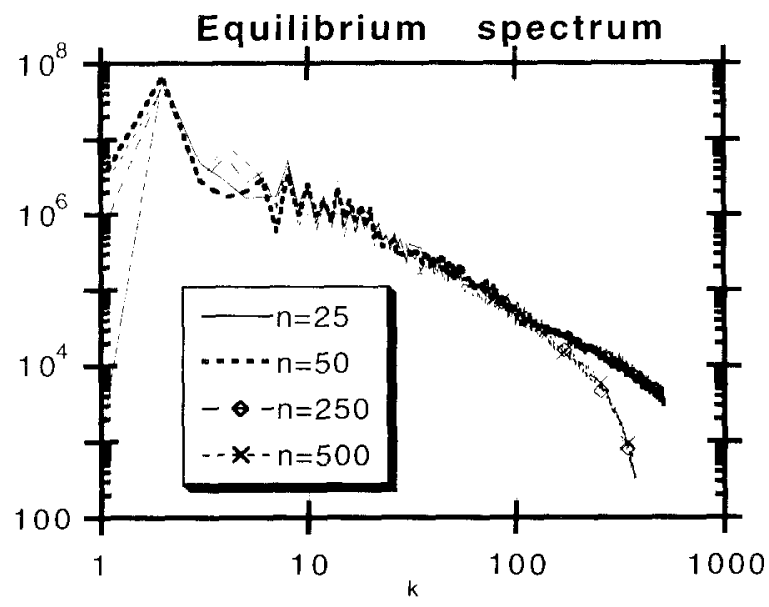

Fig. 5. Spectra for equilibrium advection-diffusion, forced with $\varepsilon=0.1$ strips. Various times. 
injection range? In Section 5 we will present some evidence that this is indeed the case. However, it will be seen that truly enormous resolutions are needed to approach the Batchelor spectrum. It seems to us a more important matter to resolve the question of how dissipation determines the anomalously steep spectrum. We raise the question, but do not yet have an answer.

The concentration PDF for the equilibrium case is shown in Fig. 6. There is a spike at $|c|=1$, arising from the forcing in the strips; the plotting of this has been suppressed to make the rest of the PDF clearer. The PDFs take longer to reach equilibrium than do the spectra, particularly for small concentrations. For $|c|>0.2$ the PDF has converged by $n=50$, but the smaller concentrations continue to evolve gradually up to about $n=250$. We cannot at this time discount the possibility that the slow evolution of the small concentration values is influenced by numerical error in our mapping scheme. At $n=250$, the PDF is very different from that prevailing in the decay case. Rather than being Gaussian, or even exponential, the PDF is characterized by a linear decay from its peak at $c=0$, tailing off to a uniform distribution for $0.67<|c|<1$. We do not suggest that all forcings will produce such dramatic discrepancies with the decay PDFs, but our results certainly show that the nature of the forcing cannot be swept under the rug when considering equilibrium concentration PDFs. Incorporating forcing into the theory of concentration PDFs will be an interesting and challenging task for the future.

A counterpoint to these results is provided by analysis of the pseudo-vorticity PDFs for the family of turbulence models simulated in Pierrehumbert et al. [23]. These are also equilibrium runs, but rather than the tracer (in this case pseudo-vorticity) values being reset to a fixed value at each time-step, only the large-scale projection is re-set, and even then the phase is randomized. This system results in PDFs (Fig. 7(a)) that are more like those prevailing in the decaying tracer case. The PDFs appear to have non-Gaussian tails. Rather remarkably, there does not seem to be any great difference in the PDFs between the spectrally local case (dominated by small-scale advection) $\alpha=1$, and the highly nonlocal case $\alpha=3$ dominated by large-scale advection. The marginally local case $\alpha=2$ does show some peculiar and erratic behavior in the tails.

Returning for the moment to the passive tracer case, we note that although the concentration fields show markedly different behavior from the decay case, the gradient PDFs (not shown) exhibit essentially the same behavior as that prevailing in the decay case. They are lognormal, and self-similar under scaling. At $n=25$ the width of the

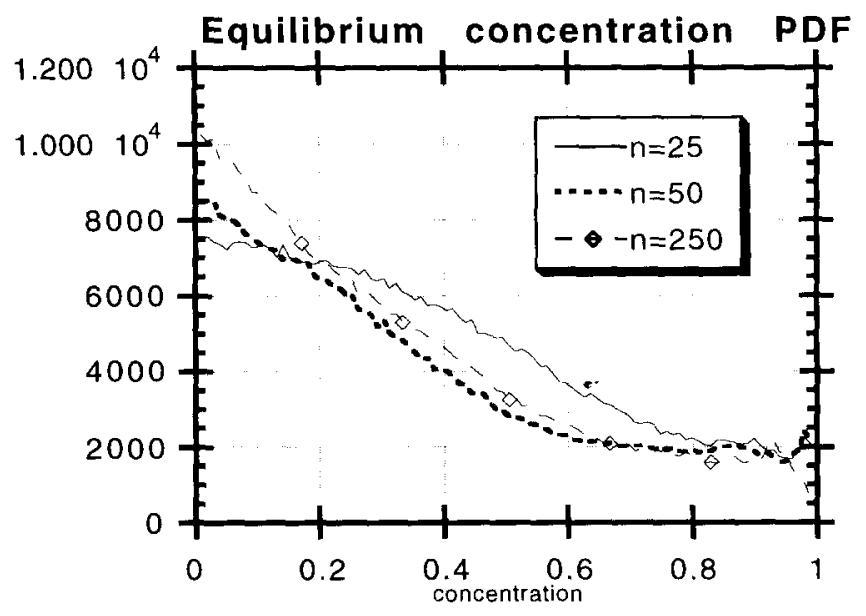

Fig. 6. Concentration PDF for equilibrium case. 
(a)

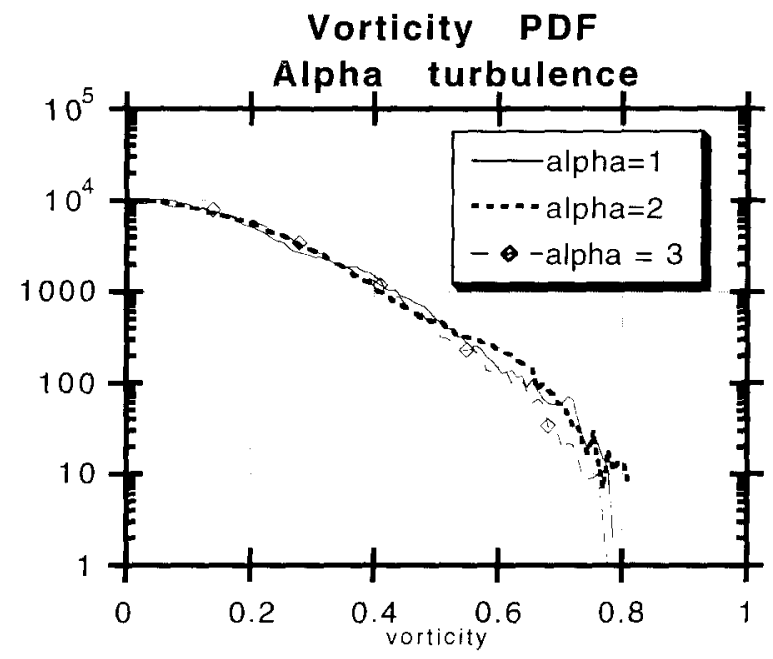

(b)

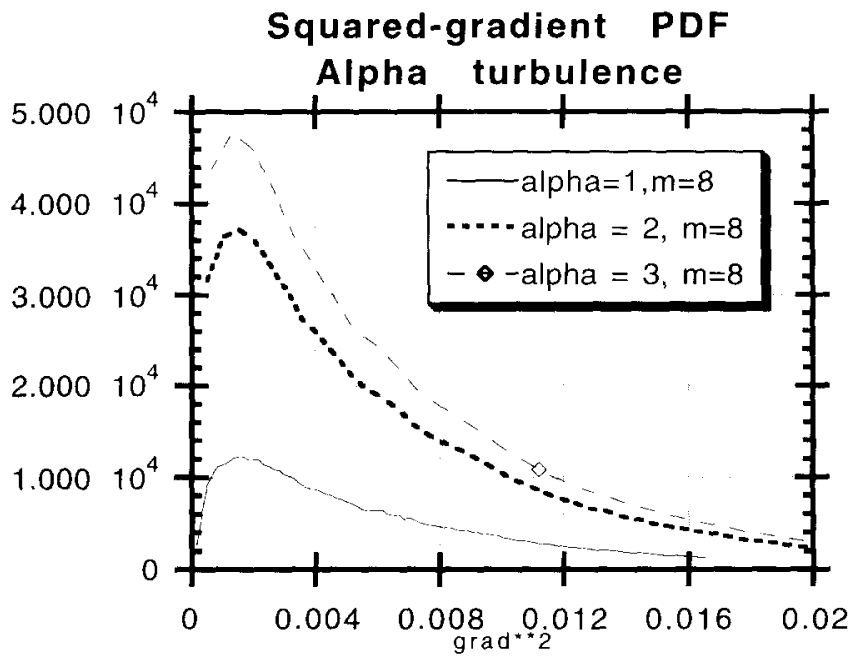

(c)

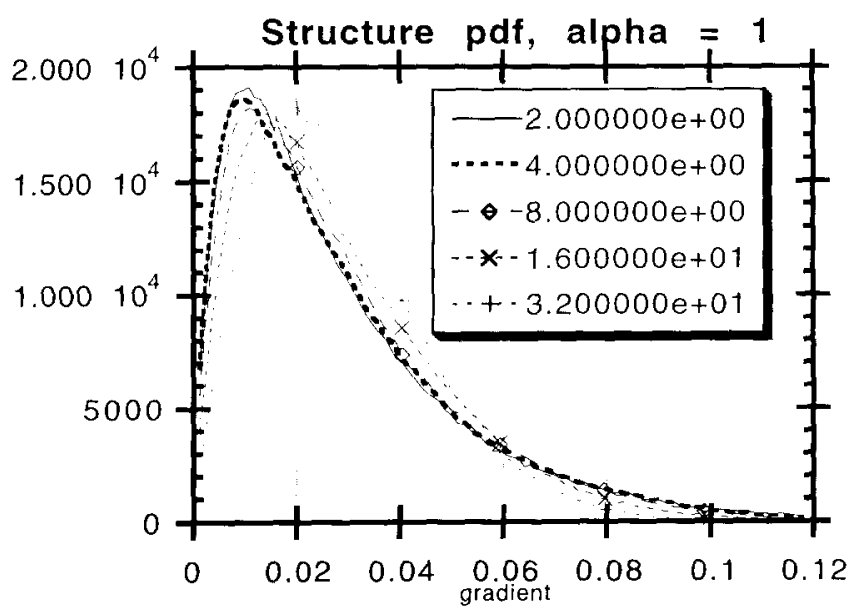

Fig. 7. PDF for 2D $\alpha$-turbulence ( $\alpha=2$ is conventional Navier-Stokes). (a) Pseudo-vorticity PDF for various $\alpha$. (b) PDF of grad ${ }^{2}$ field of pseudo-vorticity for various $\alpha$, with smoothing $m=8$. (c) Structure PDF (as in Fig. 4(c) but for pseudo-vorticity instead of concentration) for $\alpha=1$. 
distribution, $\sigma$, scales like $m^{-0.37}$, with box-size which is slightly steeper than the scaling law in the decay case. The 'structure PDFs' (i.e. with the squared-gradient computed after smoothing) also show similar shapes and scaling to those in the decay case.

The squared-gradient PDFs for the turbulence models of Pierrehumbert et al. [23] show behavior that is quite similar to those we have seen for the passive tracer case. Some representative curves are shown in Fig. 7(b). The width-scaling law for the gradient PDF is $m^{-0.34}$ for $\alpha=1, m^{-0.38}$ for $\alpha=2$, and $m^{-0.32}$ for $\alpha=3$. To within the errors of the estimate, these can all be considered the same, and the indication is that the gradient field is an FDF random field rather than an intermittent multifractal. In this case, however, we cannot discard the possibility of multifractality with as much confidence as we did in the passive tracer case. The extent of the scaling range is smaller for the turbulence models, and so the logarithmic behavior required for multifractality can arguably fit the data as well. Considerably higher resolutions will be needed to resolve this question. The substantial independence of $\alpha$ indicates that although $\alpha=1$ is formally more local spectrally than the other cases, and although the tracer filaments curdle rather than remaining lamellar (see ref. [23]), the curdling process is evidently intermittent enough that the gradient field over most of the domain is still controlled by iarge-scale processes.

The structure PDFs are shown in Fig. 7(c) for $\alpha=1$. These are rather different from what we have seen for the passive tracers. Rather than collapsing onto a single curve, the rescaled PDFs systematically change shape as $m$ is increased, and only collapse between $m=16$ and $m=32$. This is indicative of a substantially different kind of long-range order than that prevailing in the passive scalar advection problem. Other values of $\alpha$ behave similarly. This result is somewhat at odds with the previous indications that the squaredgradient field is FDF rather than multifractal, and therefore formally has $D_{q}=D$. Such a system should have self-similar ('Kolmogorovian') structure PDFs. However, this prediction is dependent on the link between $\kappa_{q}$ and $D_{q}$ and other asymptotic relations. Since the scaling associated with $D_{q}=D$ is only asymptotically valid at large $m$, the behavior of the structure PDFs may just result from the failure of asymptoticity. Again, much higher resolutions are needed to resolve the question.

The fact that none of the PDFs we have discussed is able to distinguish the obviously different tracer morphology prevailing between $\alpha=1$ and $\alpha=2$ (see Fig. 2 in ref. [23]) reveals that these tools still leave much to be desired.

\section{ULTRA-HIGH RESOLUTION UNDIFFUSED SOLUTIONS}

The question naturally arises as to whether the anomalous spectral steepness in the equilibrium case is simply due to insufficient resolution. With a full $2 \mathrm{D}$ calculation, it is unlikely that sufficient resolution will be available definitively to answer the question anytime soon. However, for undamped exactly conservative tracers, it is possible to determine the spectrum at resolutions several order of magnitudes higher. This is so because in the absence of diffusion, the concentration value on a particle is independent of that on neighboring particles. Thus, we can compute the equilibrium concentration profile $c(x)$ along a given line by running trajectories backwards for each point on the line, and determining whether they originate in the +1 forcing strip or the -1 forcing strip. Isotropy can be probed by doing the calculation for lines of various orientations.

It should be noted that even in the absence of small-scale damping, the forced problem as we have outlined it has a shortwave cutoff scale, which arises in the following manner. For forcing strips of total area $A$, the probability of a particle avoiding the forcing region for $n$ iterations is $\left(1-A /\left(4 \pi^{2}\right)\right)^{n}$, which becomes very small for $n \gg 4 \pi^{2} / A$. Thus, a 
Table 3,

\begin{tabular}{lc}
\hline$\varepsilon$ & \multicolumn{1}{c}{ Inner scale } \\
\hline 0.50000 & 0.043214 \\
0.25000 & 0.00093373 \\
0.10000 & $3.0141 \times 10^{-8}$ \\
0.0100000 & $10^{-69}$ \\
\hline
\end{tabular}

'typical' age of a parcel following its most recent resetting is $4 \pi^{2} / A$ iterations. If $\lambda$ is the typical Lyapunov exponent, the scale generated in this time from forcing strips of thickness $\varepsilon$ is $\varepsilon \exp \left(-\lambda 4 \pi^{2} / A\right)$. With $\lambda=0.5$ and the forcing geometry we have adopted, this inner length scale is given in Table 3 . The inner scale is exceedingly small (smaller in fact than the radius of an electron, for $\varepsilon=0.01$ ), but it is in fact present.

Spectra computed along the lines $x=\pi$ and $y=\pi$ were found to be practically identical at small scales, confirming that there is sufficient isotropy for spectra along a single line to be indicative of the full behavior. Spectra for the former line at various $\varepsilon$ are shown in Fig. 8 (a). These were computed based on 1048576 points, and with such an enormous range of scales the power-law scaling cannot be held in dispute. Given that the inner cutoff scale is only marginally resolved for $\varepsilon=0.1$, and is orders of magnitude below the sampling interval for $\varepsilon=0.01$ and $\varepsilon=0.001$, error due to aliasing is a prime concern. For $\varepsilon=0.001$, the spectrum is entirely flat; because of undersampling, all information about spatial correlation is lost and the signal is indistinguishable from white noise. For $\varepsilon=0.01$, the white noise spectrum due to aliasing is visible at short wavelengths, but there is indication of a power-law spectrum at somewhat longer waves. The spectral slope of this range is approximately $k^{-0.6}$. Given that the power in this spectrum is highly divergent, however, one must be suspicious that it, too, is an artifact of aliasing. This is confirmed by examining spectra computed from signals subsampled by a factor of 16 and 64, and also by recomputing spectra for the full 1048576 points arranged on a segment of length 0.001 the original one. Both spectra (not shown) indicate that the $\varepsilon=0.01$ spectra in Fig. 8(a) are strongly influenced by unresolved small scales.

Finally, for $\varepsilon=0.1$, a $k^{-1}$ Batchelor spectrum is obtained. Given that $k^{-1}$ is divergent, one must still be wary of aliasing in this case. However, the fact that the inner scale is only slightly shorter than the sampling interval partly allays this fear. Further, in Fig. 8(b) we compare the spectra for the full record with spectra computed from subsampling by a factor of 16 and 64. It appears that the aliasing error reliably moves to short waves as the sampling increases. From this we conclude that the Batchelor spectrum is indeed realizable given an extensive enough inertial range. The results suggest that extremely high resolutions are needed to recover the spectrum, however.

As $\varepsilon$ is increased beyond 0.1 , the inner scale increases and moves into the range of the resolved scales, compressing the 'inertial' range. When this happens, the spectra begin to be influenced by the detailed physics determining the statistics of the inner scale. In fact, there is not really a unique 'inner scale' but rather a spectrum of 'inner scales' associated with the spread of Lyapunov exponents and the spread of times taken before an orbit encounters a resetting region. In the limit of an inner scale that is sharply bounded below, one would recover the $k^{-2}$ jump spectrum as in the theory of Saffman [22]. Small-scale events are sufficiently probable to render the spectra shallower than Saffman's theory, but steeper than $k^{-1}$. For $\varepsilon=0.25$, the spectral shape is $k^{-1.25}$, and for $\varepsilon=0.5$ the shape is $k^{-1.52}$. 
(a)

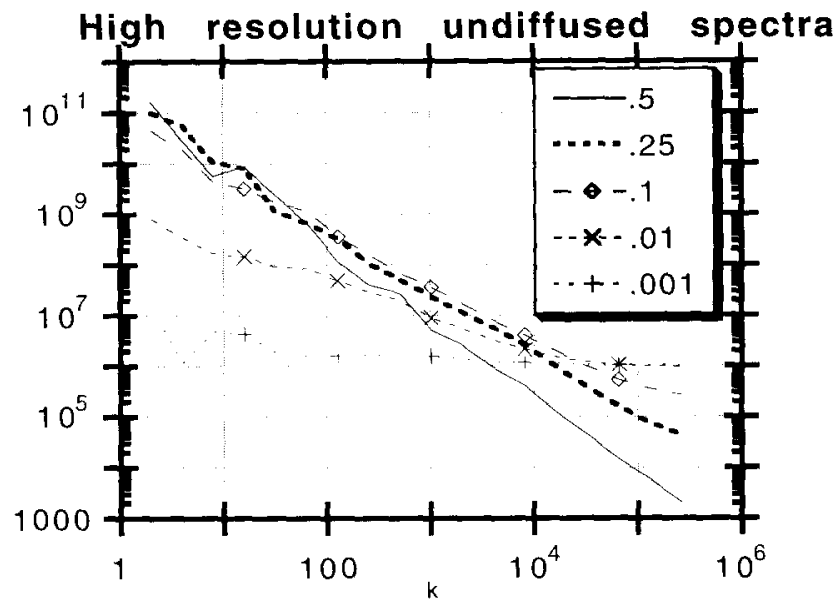

(b)

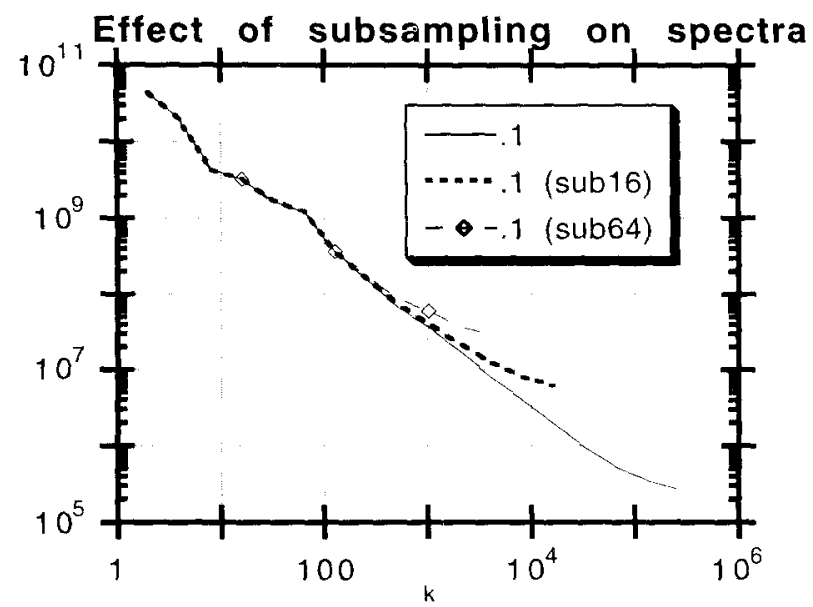

Fig. 8. (a) High-resolution (1048576 point spectra) for undamped equilibrium case with various forcing widths $\varepsilon$. (b) Effect of subsampling for $\varepsilon=0.1$, showing localization of aliasing errors at high wavenumbers.

\section{CONCLUSIONS}

We have outlined a general computationally tractable approach for addressing a range of problems concerning tracer microstructure created by large-scale advection and small-scale diffusion, through the use of iterated maps. Using this technique, we have studied spectra, concentration PDFs, multifractal scaling of squared-gradients, and cancellation exponents. Results for the idealized system have been compared and contrasted with similar diagnostics applied to a family of fully nonlinear two-dimensional turbulence models. While more questions have been raised than have been resolved, a few clear points have at least emerged.

For the freely decaying advection-diffusion problem, there is evidence that the evolution settles into a 'strange eigenmode' which has arbitrarily fine-scaled variability limited only by diffusion, but which is statistically self-similar in time. The variance decays exponentially in time, with a rate governed by the dominant Lyapunov exponent, and independent of diffusivity. The concentration power spectrum for this eigenmode is not algebraic, but 
rather exhibits a broad exponential rolloff. The concentration PDFs are self-similar in time, and are approximately Gaussian for small concentrations but have exponential tails for extreme concentration excursions. These PDFs appear to conform well to the theory of Sinai and Yakhot [7]. It is clear from these results that a Batchelor $k^{-1}$ spectrum is not the long-term solution for the freely evolving case in the presence of dissipation. The 'strange eigenmode' is the correct end-state.

A very different picture is obtained when the variance is maintained against decay by periodically reintroducing unit-magnitude concentration in certain strips at large scales. In this case, the spectrum is indeed algebraic, though it is significantly steeper than $k^{-1}$. It is significant that, at similar resolutions, the pseudo-vorticity spectra for the large-eddy dominated cases $\alpha=2$ and $\alpha=3$ in the 2D turbulence models of Pierrehumbert et al. [23] show the same kind of anomalous steepness. We suggest that steep spectra of this sort are characteristic of mixing by large-scale advection and small-scale diffusion, at least until truly enormous resolutions (perhaps $10^{6} \times 10^{6}$ ) in $2 \mathrm{D}$. An undiffused simulation at the latter extremely high resolution indeed shows a $k^{-1}$ spectrum when aliasing problems are surmounted and when the non diffusive concentration microscale (which we have defined above) is sufficiently far removed from the inertial range. There is as yet no quantitative theory of the diffusively influenced steep spectra prevailing in the lower resolution calculations.

The PDFs of concentration in the equilibrium case are markedly different from those in the freely decaying case. Apart from a spike at the concentration value introduced by the forcing, these distributions are much more uniform than those in the decaying problem. This indicates that theories of the type of Sinai and Yakhot do not always work in the presence of variance maintenance by large-scale forcing. In contrast, though, the equilibrium pseudo-vorticity PDFs for the turbulence models of Pierrehumbert et al. [23] do show a behavior that is more like the Sinai-Yakhot theory, though the range of Gaussian behavior is broader and the exponential tails are less pronounced.

A robust result we have found throughout all the models we have examined-decaying tracer, equilibrium tracer and ' $\alpha$-turbulence' (Pierrehumbert et al. [23])-is that the squared-gradient field is a 'fractal degree of freedom' random field rather than a multifractal. We have argued that the scaling of the width of the lognormal distribution of the squared gradient under smoothing is anomalous, and reflects the underlying geometry. The lognormal behavior of the squared-gradient PDF is closely related to the Gaussian distribution of Lyapunov exponents, which is known for the advection-diffusion problem, and which we conjecture for the $\alpha$-turbulence problems.

We have also made a foray into the subject of cancellation exponents, by examining the PDFs of the magnitude of the gradient computed after smoothing is first applied to the concentration (or pseudo-vorticity) field. For both the decaying and equilibrium passive tracer case, the concentration structure PDFs for various degrees of smoothing are 'Kolmogorovian,' in the sense that they are self-similar under rescaling by the mean gradient. It was argued that this is indicative of a non-intermittent squared-gradient field. We gave a concrete realization of the $k^{-1}$ Batchelor spectrum in terms of a nonintermittent space-filling random gradient model. However, it was also shown that the Batchelor spectrum is perfectly compatible with intermittent models as well, because of the leeway provided by varying the degree of cancellation between positive and negative gradient regions.

The structure PDFs are less self-similar for the $\alpha$-turbulence cases and suggest that (relative to the typical gradient at each scale) weak gradients are more probable at small scales then they are at large scales, as if localized weak-gradient regions are systematically eaten up by neighboring same-signed high gradients when the field is smoothed. Most of 
our results concerning $\alpha$-turbulence should be regarded as tentative, though, owing to a marginally resolved inertial range.

Under what situations would behavior of the sort we have seen above be expected to prevail? It comes down to a question of whether the advecting field has in some sense a dominant timescale, and the existence of such a timescale can be probed by examination of the finite-time Lyapunov exponents for the trajectory problem arising from a given velocity field. For the iterated map we have studied, these exponents are well-behaved by construction. For a turbulent velocity field with small scales, however, the Lyapunov exponents could be highly dependent on the dissipative cutoff. Notably, for a $k^{-5 / 3}$ energy spectrum, the associated velocity field has divergent gradients. In principle, then, the Lyapunov exponents could become divergent as resolution is increased. This need not happen, however, if the correlation time of high-gradient regions is sufficiently short. Indeed, this appears to happen for atmospheric winds simulated by a general circulation model, for which case the trajectory problem has a well-defined exponent [5]. The velocity field for the $\alpha=1$ turbulence model discussed in Pierrehumbert et al. [23] also has divergent gradients. While we have not computed Lyapunov exponents yet for this case, the PDFs of the squared-gradient field are strongly suggestive of behavior similar to that of the iterated map. Coming to an understanding of finite-time Lyapunov exponent distributions for trajectory problems arising from turbulent velocity fields is, in any event, of prime importance. The $\alpha=1$ model provides a good forum for probing the relative role of large-scale and small-scale advection, and would be a good starting point for such a study. Simulations of passive tracer mixing by the velocity field of this model would provide invaluable complementary information.

Acknowledgements - The advection/diffusion calculation reported here grew out of a stimulating discussion with Leo Kadanoff and Itamar Procaccia. Discussions with Samuel Vainshtein and Yunson Du were indispensible in clarifying the connection between cancellation exponents, structure functions, and generalized dimensions. My thinking about structure functions has also been much influenced by discussions with Alexander Marshak and Anthony Davis. The results in Section 5 were stimulated by exploratory calculations carried out by Yannis Antoniades. This work was supported by the National Science Foundation, under grant ATM 89-20589.

\section{REFERENCES}

1. G. K. Batchelor, Small-scale variation of convected quantities like temperature in turbulent fluid-Part 1: General discussion and the case of small conductivity, J. Fluid Mech. 5, 113-133 (1959).

2. R. T. Pierrehumbert, Chaotic mixing of tracer and vorticity by modulated travelling Rossby waves, Geophys. Astrophys. Fluid Dynamics 58, 285-319 (1991).

3. R. T. Pierrehumbert, Large-scale horizontal mixing in planetary atmospheres, Phys. Fluids A3, 1250-1260 (1991).

4. R, T. Pierrehumbert, Spectra of tracer distributions: A geometric approach, in Nonlinear Phenomena in Atmospheres and Oceans, edited by R. Pierrehumbert and G. Carnevale. Springer, New York (1992).

5. R. T. Pierrehumbert and H. Yang, Global chaotic mixing on isentropic surfaces, J. Atmos. Sci. 50, 2462-2480 (1993).

6. R. R. Prasad, C. Meneveau and K. R. Sreenivasan, Multifractal nature of the dissipation field of passive scalars in fully turbulent flows, Phys. Rev. Lett. 61, 74-77 (1988).

7. Ya. G. Sinai and V. Yakhot, Limiting probability distributions of a passive scalar in a random velocity field, Phys. Rev. Lett. 63, 1962-1964 (1989).

8. T. Horita, H. Hiroki, I. Ishizaki and H. Mori, Long-time correlations and expansion-rate spectra of chaos in hamiltonian systems, Prog. Theor. Phys. 83, 1065-1070 (1990).

9. P. Grassberger, R. Badii and A. Politi, Scaling laws for invariant measures on hyperbolic and nonhyperbolic attractors, J. Stat. Phys, 51, 135-178 (1988).

10. E. Ott, Y. Du, K. R. Sreenivasan, A. Juneja and A. K. Suri, Sign-singular measures: Fast magnetic dynamos, and high Reynolds number fluid turbulence, Phys. Rev. Lett. 69, 2654-2656 (1992).

11. Y. Du and E. Ott, Fractal dimensions of fast dynamo magnetic fields, Physica D, 67, $387-417$ (1993).

12. B. J. Bayley, Infinitely conducting dynamos and other horrible eigenproblems, in Nonlinear Phenomena in Atmospheres and Oceans, edited by R. Pierrehumbert and G. Carnevale. Springer, New York (1992).

13. E. Ching, Probability densities of turbulent temperature fluctuations, Phys. Rev. Lett. 70, 283-286 (1993).

14. A. Pumir, B. Schraiman and E. D. Siggia, Phys. Rev. Lett. 66, 2984-2986 (1991). 
15. F. Varosi, T. M. Antonsen and E. Ott, The spectrum of fractal dimensions of passively convected scalar gradients in chaotic fluid flows, Phys. Fluids A3, 1017-1028 (1991).

16. E. Aurell, U. Frisch, J. Lutsko and M. Vergassola, On the multifractal properties of the energy dissipation derived from turbulence data, J. Fluid Mech. 238, 467-486 (1992).

17. Y. Du, T. Tel and E. Ott, Characterization of sign-singular measures, Physica D, in press (1994).

18. A. Marshak, A. Davis, R. Cahalan and W. Wiscombe, Bounded cascade models as non-stationary multifractals. Phys. Rev. E, 49, 55-79 (1994).

19. A. Davis, A. Marshak, W. Wiscombe and R. Cahalan, Multifractal characterizations of non-stationarity and intermittency in geophysical fields, observed, retrieved or simulated, J. Geophys. Res. 99, 8055-8072 (1994).

20. S. I. Vainshtein, Y. Du and K. R. Sreenivasan, Sign-singular measure and its association with turbulent scalings, Phys. Rev. E, 49, 2521-2523.

21. Y. Tessier, S. Lovejoy and D. Schertzer, Universal multifractals: Theory and observations for rain and clouds, J. Appl. Meteor. 32, 223-250 (1993).

22. P. G. Saffman, On the spectrum and decay of random two-dimensional vorticity distributions at large Reynolds number, Stud. Appl. Math. 50, 377-383 (1971).

23. R. T. Pierrehumbert, I. Held and K. Swanson, Spectra of local and nonlocal turbulence in two dimensions. Chaos, Solitons \& Fractals, to appear (1994).

24. T. M. Antonson and E. Ott, Fractal measures of passively convected vector fields and scalar gradients in chaotic fluid flow, Phys. Rev. A39, 3660-3671 (1989). 